\title{
Notes on a common beat
}

\author{
Alessandro Capurso \# \\ $\%$ in 8 (i)
}

\begin{abstract}
The following essay would like to elaborate on a possible "philosophy of efficiency" connected to the contribution "Implementation model of a universe based on information" (a technical paper from the same author proposing a framework for Quantum Gravity, available at doi.org/10.31219/osf.io/9a26r). The insights derived from the interpretation of spacetime considered focus on causality, free will and efficiency, promoting collaboration, empathy and trust. Even if some of the concepts presented are common to several cultures at different levels of abstraction, this paper does not pretend to have scientific value nor suppose any reference to religion. The scope is just to draft a high level parallel between the description of the Universe proposed in the scientific contribution and a more philosophical interpretation of life, inspired by efficiency and based on the memory of a common beat.
\end{abstract}

Keywords: time; free will; thick present; memory; consciousness; efficiency; sociology;

\section{INDEX}

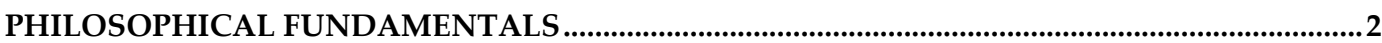

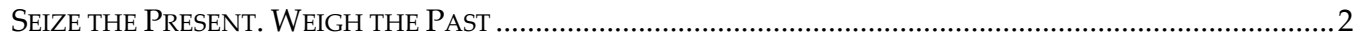

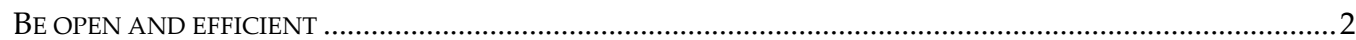



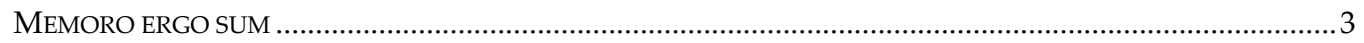





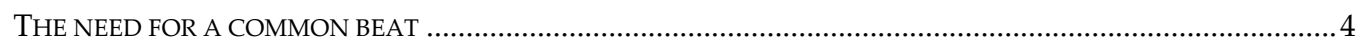

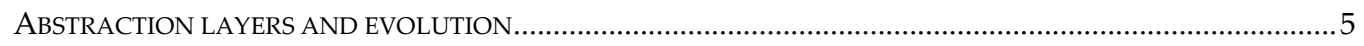

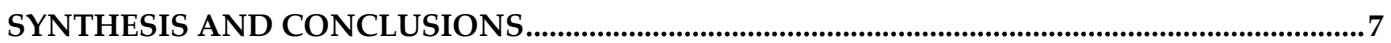



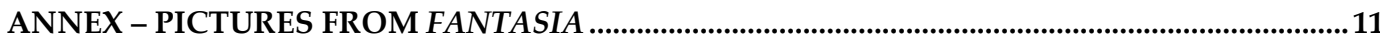

\footnotetext{
\# ale.capurso@gmail.com
} 


\section{Philosophical fundamentals}

\section{Seize the Present. Weigh the Past}

The interpretation of the nature of time has seen two opposite descriptions. The first, called Eternalism, is often related to the theory of Relativity and consider an ever-existing time, real in both the past and the future, and of which we perceive only limited cones of information. The opposite perspective, known as Presentism in physical theories, is connected to the idea of evolution itself, and pictures time as everchanging: a vanishing present compressed between past and future instants. The focus is on the difference between the past, already happened and irreversible, and the future, still undefined and open. The axis of time seems to become real only in the current instant and in the causal consequences of the past ones. The interpretations of Quantum Mechanics (QM), in an ironic and iconic uncertainty, stand on both sides of the diatribe. There are timeless formulations (where the variable $t$ disappears in the equations), resulting in an "eternal picture" of what is possible, as well as interpretations that focus on information evolution and on the irreversibility of events, closer to Presentism.

Beside physics, latest developments in neuroscience point to a perception of time in our brain connected to a "thick present". Our neurons elaborate different asynchronous stimuli and derive a coherent picture of the surrounding space in each "perceived instant". Our perception is not always physically accurate but to our brain looks coherent and this is enough for a perception of a "flowing time": a past connected to the future through what is happening in the present. Many oriental and classical philosophies as well consider only the present as real, promoting the focus on each moment as a gift full of opportunities.

The physical interpretation derived by the author favors an evolving picture of information and considers time as real only in the current instant, causally affected by what happened and open in the future possibilities. Causality allows the influencing of future, giving meaning to the freedom of action in the present potential. Following those ideas, we should weigh the past and seize the present, projecting towards better futures through the choices we are presented and accepting whatever else comes as out of our control.

\section{Be open and efficient}

If the concept of time is connected to the idea of evolution and to the changes in the information, space could be connected to the changing information itself. We might ask what "information in space" is changing and, given the present instant as the only reality in time, wonder about the meaning of the reality of space. In the considered model, the concept of "imaginary distances" (as an imaginary time from the point of view of photons) helps defining the information in space. Within each real present instant, a network among imaginary points of weighted information of possible distances could be seen as the ingredient needed to represent all space. The term imaginary should not be intended as "non existing", in opposition to real, but as an orthogonal information and, eventually, as an imaginary axis of spatial distances in a complex spacetime.

If distance is considered as the elementary information in space, described as imaginary and probabilistic, we could ask if spacetime behavior could give any insight for an efficient "management" of such an enormous quantity of information and for the search of the optimal variety in the probabilistic evolution. In the physical interpretation proposed, we have considered efficiency in the compression of such information thanks to a logarithmic perspective on spacetime, able to highlight the different scales of phenomena. The right sizing of the information through an analysis at different scales could offer the most accurate synthesis. The maximum compression of information leads to maximum entropy. The concept of entropy carries the meaning of both "summarized information" and of "apparent randomness" in the derived information. In each present instant, the maximum synthesis of the imaginary distances seems no useful information, but it could also be the most random seed, to be used in the computation of the imaginary possibilities in the current cycle. A random seed could computationally allow a probabilistic emergence of the imaginary information in line with QM predictions. 
The given spacetime description seems to promote efficiency in both the compression and the open evaluation of the evolving information. It considers each event and gradient at the right scale, efficiently sizing the phenomena for the highest compression ratios. Furthermore, it leverages on the maximum achieved entropy for an optimal evolution based on quantum randomness. Given the known past and the undetermined information hidden in the present potential, spacetime optimal randomness in the seed for the computation of evolution inspires to consider the freest imagination in the evaluation of the future steps, with an always open perspective.

Eventually, the model of spacetime considered seems to suggest the wildest openness in the imagination of possibilities and, at the same time, efficiency in the elaboration their causal weight, learning from experience and properly sizing every information, in both space and time, evolving coherently on the causality of our choices.

\section{Ontological perspectives}

\section{Memoro ergo sum}

In the proposed scientific description of spacetime, an absolute perspective on the passage of time is derived considering a frame of reference at infinity in space. This allows the identification of a global "thick computational present" that accounts for the causal set of past events. The information of the past is connected to gravity. The information of gravity is then considered as the "proof of existence" of a mass-energy somewhere in space during the previous instant. The thick present information (from $2 k T-1$ to $+2 k T+1$ ) persists in a logical consistent memory that becomes ontological, as spacetime is considered emerging from this fundamental potential, called Absolute Time, at each present cycle $k$. Following Freeman Dyson in his "Thought Experiments in Honor of John Archibald Wheeler", the only role of the observer is the distinction between a classical past and a still open and probabilistic future, but the connection between memory and existence could be raised to even higher levels of abstraction, including human consciousness.

In medical records of people under anesthesia, there are several examples showing a connection between states of consciousness and a sense of "present awareness". In both neurological studies and several philosophical interpretations, it is the perception of a coherent unity with the present surrounding reality that allows "presence" and, eventually, Self-consciousness.

We could picture neurons connecting the already known past with the asynchronous stimuli from the surroundings (equalized on a thick present or "coherent now") and, from this coherently weighted combination, build up a logical consistent picture of reality, different from the Self and in which the Self is consciously perceived. It is then the logical consistent access to a memory where the causal past is real and the current stimuli could be equalized and openly evaluated that creates the awareness of the present and, eventually, the consciousness of the Self as an existing and persisting entity in time.

In an ontological discussion, we could answer Cartesio that, as a memory is needed to evaluate any possible thought or observation, "I have a memory with logical consistent information up to the immediate past instant, therefore I am in the present"... or just briefly, memoro ergo sum.

Note that the identification of the "memory" of the Self has to be referred at the proper level of abstraction, as further elaborated in the coming chapters. Moreover, human consciousness has nothing to do with "physical reality" or "existence in the Universe", which are a priory of human beings. Events occur and matter exists, and both are causally real, even if there are no human observers, as everything is coherent from a gravitational perspective and the Absolute Time memory is always consistent with events and causality, as the ultimate impartial bookkeeper. 


\section{Everything is interconnected}

In the proposed model of the Universe, there are non-local connections that could be pictured as spacetime wormholes (tunnel in the information space, called ER) and are related to the entanglement (called EPR, as a correlation among distant elements originated in a past event). The information of correlation shall always be globally coherent. A logical consistency check (within the computation cycle) could be implemented with a closed time-like curve $(C T C)$ in the thick present, resulting in a memory-loop of persisting potential in the Absolute Time (CTC represents an open potential as events cannot be defined on their path and loops are the most basic circuits for memory allocation also in Information Science). We can then derive a conceptual equation on the link between space and information considering $E R=E P R=C T C$ and describe the spacetime information potential through the information of these memory-loops.

What all these scientific descriptions have to do with any philosophical interpretation? Simply, that everything is interconnected through a common fundamental memory and that existence (as persistence in time) relies on the evolution of these connections and loops as a network. The records of the correlations realized in the past allow the imagination of the current potential as a network of old and possibly new connections. As time passes, reality consolidates in the memory, following the causality of events.

Extending to existing concepts, the Absolute Time memory could be related to the Sanskrit word अपरिगण्यः - apariganya. It could be seen as the cipher of the spacetime information, holding the current most detailed description of "what had been, is being and could be" since the birth of the universe. It is incalculable inside the inner relativistic and relational universe by definition, as a far seed of randomness at infinity. It is actually a "non-entity" or a nobody and eventually the everything interconnecting the everywhere.

The description of phenomena as evolving networks of information could be extended to human relations as well, as with no connections nor relative confrontation, it would be difficult to give a definition of any Self. Both the memories of emotions and of a social experience create a network of connections at a level of abstraction that we could call "Ego", and in which individuals feel defined. We are always bounded to others, in both the past and the present, and, in these bonds, we build our future. When we miss their value, we become egocentric, like a small black hole disconnected from the surrounding humans and living with chosen absolute references in its own imaginary time.

The questions of how everything could be interconnected at the most fundamental level and what is really needed to create connections and allow relative confrontation, consistent memories and, eventually, existence at any level of abstraction, are still to be properly investigated and will be left to the coming chapters.

\section{Memories of the firsTk1ss}

\section{The need for a common beat}

In the proposed model of spacetime, the correlations derive from relativistic and relational confrontation. To assure a local coherence and a global logical consistency of the information of correlation in the memory of the Absolute Time, the confrontations have to occur with absolute global references.

We could consider the Planck constant $\hbar$ as the simplest reference needed to properly define a relative confrontation in spacetime (as reminded by the QM uncertainty principle). It is an absolute reference for a quantum of angular momentum and of action, derived from the Planck units of mass, length and time, like a common beat known a priory in every point in the Universe. In the proposed model, the reference $\hbar$ depends on the characteristics of the Absolute Time memory. It is defined in the collapse of the parent Black Hole (firsTk1ss), equivalent to the inflation era of the inner imaginary universe. It is the first memory and the universal beat towards which every persisting information should be relative, as the sharpness and resolution of spacetime. 
Particles, in this context, are wavelets in the spacetime fabric. They are described as correlated information of possible emerging positions in the imaginary space (as expressed in the wave function) and show a coherent "momentum in time" (connected to the mass). The ensemble of the points of possible emergence in space could be seen as a "volume of imaginary space on a common beat" in respect to the absolute beat of reference.

The same reasoning on the needed ingredients to define a connection could be extended, from the most fundamental elements of spacetime, to other level of abstractions. Connections in any network could be seen as the sharing of a common information, phase or beat, like a kind of "shared key" among the network-nodes in respect to a known global reference or code. The common beat is related to forces of persistence inside the network, like looking for the interconnecting root and common memories.

In this context, we could then consider the concept of "transformation" as connected to the chances of integrating new information, developing new interconnecting-codes and becoming a wider network, while evolution could be intended as the alternation of persistence and transformation cycles, towards a more resilient and wider potential.

A possible example of a "common beat" could be found in Biology, described through the story of DNA, a memory and a genetic code. The network nodes identified in the "Self" could be all the cells with the same DNA, like a trusted shared information.

Considering human consciousness, we can associate the idea of a "common beat" to the concept of empathy, intended as "feeling the same". Empathy could be the correlation code at the human Ego level of abstraction, assuring strong and fair connections in our societies, and could be extended to the relation with other living beings, as sailors on the same ship. The states of "higher consciousness" (alike in several cultures throughout history) are often described as instants of "illumination", able to open the view to deeper understandings, towards a feeling of wholeness, empathy and connection with the entire Universe. Given the proposed interpretation, they could be ideally related to a brief perception of the universe common beat, as being totally in the present and perceiving everything in an instant as from infinity, following the memories of the firsTk1ss.

\section{Abstraction layers and evolution}

Every phenomenon could be described at a different level of abstraction, depending on the type of information and messages available at the given level. Biological processes, for example, can be seen from a chemical point of view (through a "communication" based on covalent or ionic bonds) or through QED (focusing on the Electron field and on the exchanged virtual photons). It depends on the "scale of the information" that we are considering in our representation of reality and the desired precision. These models are anyway simplifications of the phenomena, focused on a given agreed framework, as every layer is an abstraction of an interconnected and global coherence.

In the search for new scientific theories, it is common to follow a "reductionist" approach towards the most fundamental description. The proposed model focuses on the elementary scale and considers spacetime as a network of imaginary points connected through links of correlation. The connection occurs through the simplest correlation code: a common beat among the imaginary distant points in respect to a global beat of reference of the Universe. Elementary particles are, in this context, networks of correlated points (on a common beat) within a greater network (the Universe).

A model of networking based on information and communication messages could be applied at any level of abstraction, including human societies. All human groups (Religions, States, Companies, ...) exchange "information", "energy" or "work" (that could be knowledge, resources, time, money or any other physical or abstract concept) and communicate creating a network. The "interconnecting code" might have no relation with any physical reality and could be freely chosen and agreed among the members of the group: it works as long as it is a "shared key" of communication and link. The ability to imagine its own codes of interconnection beyond physicality allowed the homo sapiens species to flourish and eventually dominate the other forms of life on Earth. 
The communication code, in its associated "imaginary value", mostly defines the network and its evolution. Cash is worth something only because humans give to the specific pieces of paper an agreed "imaginary value" and the sum of all the lent and received money makes the economic network. On a similar line, a sacred book has "value" only to the believers of the specific cult or religion and becomes a "connecting code" among them, assuring common values, vision and "momentum in time", as long as it is believed.

We could choose any imaginary messenger and equivalent level of abstraction, still it is worth asking if there are any best practice that we can learn from the most basic network. As the end goal always seems to be an "optimal persistence", we should consider the insights we could get from the most fundamental network in terms of "survival strategies". We should consider both the weaknesses "inside" the network, as well as the limits of a given network in relation with the greater environment in which the network exists. Moreover, we should consider forces of persistence, connected to the "strength" (or agreed value) of the common code and the resilience of the shared information, as well as of transformation, related to the ability to integrate new information and use new codes.

Starting from what happens inside the network, natural systems seem to enjoy gradients and divergences in the search for optimal evolution, but tend to avoid very high local concentrations of "extra energy" (as not stably persisting in a single entity), quickly diffusing the overload through the interconnected elements. It could be temperature, energy, money, knowledge or any other agreed imaginary code, but strong unbalances should be avoided, "smoothing the peaks" of local concentrations in exponential time. Eventually, given the limited efficiency achieved by single entities, a localized high concentration of any "extra potential" managed by few nodes only would prevent the realization of the full network potential, missing the synergies of the greater structure.

A cause of these inefficiencies could be related to a "weakness" of the interconnection among the neighbor nodes. The weakness could be due to the absence of local connections (no communication) or the lack of a "node equivalence" (fair sharing of resources/load). These local inefficiencies might lead to a weakness of the whole network and, as everything is interconnected, in the long term become a weakness for the local unbalanced nodes too.

These concepts could be easily applied to human societies, where it is common to see unbalanced distributions of wealth and opportunities that often lead to inefficiencies. Usually, the unbalances are due to the lack of empathy and trust, as well as to the lack of a true "equivalence" among human beings. Beyond the equivalence on basic rights (that should be common to all forms of life and still is missing in some human societies), possible examples of inequalities could be found in salaries, where there are several orders of magnitude in the value of the time of different people, even for the same kind of effort ${ }^{1}$. Salaries are just an example of how human beings divide themselves in economic groups with different access to goods and rights, pretending it is all just a matter of merit. Considering a random availability of potential among newborns, limiting the chances of education depending on the economic status of the group to which the newborns belong prevents the development of their potential, and eventually limits the development of the entire group. It may even seem that wealthier groups, in a fear of loss, often may limit the potential developing in other human groups to keep the status quo.

As everything is interdependent and relative in a global system, also the behavior of the network within the greater context determines its persistence in time. At the scale of networks, as at the scale of individual nodes, it is often a matter of equilibrium of resources between what is needed "inside" and what is still available "outside".

\footnotetext{
${ }^{1}$ A farmer in a poor country might get paid few dollars a day and have no access to public health, while a farmer of a first-world economy could easily earn 2 orders of magnitude more, a top manager 3 and world known actors, athletes or CEO, even 5 or 6 . Wouldn't 2 to 6 times already be enough for a gradient in human evaluation? Is it fair to consider multiplication by hundreds, thousands and more? Probably, a logarithmic progression (as the perspective on different spatial scales used in the Absolute Time memory) could be a more balanced synthesis of the "produced value" for human beings as well.
} 
From this perspective, human societies have often proved very greedy and disconnected from the surrounding environment. As a symptom of the disequilibrium of the human network within the planet biosphere (beyond the biodiversity decimation), the global warming became in the last decades no more a question of efficiency but of survival chances and eventually of human persistence. Planet Earth will exist anyway, and the biosphere may eventually recover (despite our hard prints), but there could be no more "human observers" wandering about their consciousness.

It is crucial to note that both "internal inefficiencies" and "external unbalances" seem connected to the will of storage of resources (as if it were "a matter of size" and we could "always need more") and to the belief in a non-collaborative human-network (lack of trust and empathy). It seems human beings underestimate as well the costs of their supposed "right to accumulate", for both the environment and the global human network².

From a perspective based on efficiency and natural selection, it really looks nonsense and shortsighted. We should focus more on what connects and makes equal every human being (even of the coming generations). Moreover, we should promote a transformation in the interpretation of our role as individuals, widening our perspectives and our knowledge for the most open and efficient collaboration.

Considering global warming as one of the most relevant threat to human persistence beside our own selfishness, it is probably worth investigating as well if new technologies for energy production and storage could be derived considering static mass information and CTC-bonds as a possible resource.

A global scientific collaboration towards a "quantum energy" from the decoherence of massive particles (through the interaction with the nodes and links at the most fundamental level) could probably help addressing at least part of the problem. If, as humanity, we do not evolve to collaborate at a global level and feel part of the Earth biosphere, even a new form of energy would only delay our farewell.

\section{Synthesis and Conclusions}

We have proposed a parallel between a scientific description of the Universe at a fundamental level and a possible philosophy inspired by the same characteristics.

We have described spacetime as an interconnected network of information, which is related to the memory of past events and evolves on a $2 T$ elaboration cycle (thick present). Space (described through imaginary distances) is pictured as emerging from the links of correlation potential in each present instant. These links are considered the fundamental information to describe spacetime and are derived from the confrontation of a proper momentum in time (or "common beat", connected to the entanglement among correlated imaginary points and the mass) with a global beat of reference.

Both persistence in the spacetime memory as well as emergence in the imaginary space occur with maximum efficiency. The information of the imaginary spatial distances is compressed to the limit thanks to a wavelet analysis at different scales and the corresponding maximum entropy achieved from the compression is used, in each cycle, as an optimal seed for random processes in the computation of space at the quantum scale.

Starting from a Presentism perspective on time inspired by efficiency, we have concluded that we should focus on the current instant, and avoid following far imaginary needs, as possibilities and consciousness exist in the present awareness and in the memories of the past events.

\footnotetext{
${ }^{2}$ As a possible example, we can consider the inefficiencies hidden in the "food economy": the wasted supplies in developed countries could easily feed more than once the entire human population and the intensive production of crops and meat impoverishes the soil and has already destroyed several natural ecosystems.
} 
In each instant, it is crucial to have a momentum that identifies our intentions, our memories and eventually our being. In each instant, we should maximize the possibilities, being open and rich in the imagination, as any possible reality had been firstly imagined in some memory. Furthermore, we should maximize the experience from the past, understanding the causal consequences of events through a perspective at different scales, beyond the limits of our narrow perception. We consider phenomena focusing on days, months and at most few years, but we miss both the present instant as well as the perspective of a lifetime. Blindly we forget mistakes and experiences of the past generations and miss the relevance of the causal consequences of our choices in 100 years. We should be very well aware that, as last century wars and discoveries shaped our today society, most of the reality of 2121 is being imagined and it is already becoming real today.

In the proposed model, everything could be described as a network and, at the most fundamental level, all the Universe is interconnected in the causal memory of time.

The concept of memory (to store the causal past and elaborate the present potential) becomes ontological: a requirement for existence and persistence. A common beat in respect to a global reference (as a shared code among the network nodes) is given as the main ingredient to allow interconnections.

In the context of networks, the efficiencies of the internal structure (in terms of the reliability of the connections), as well as and the sustainability and the integration with the external context, determine the evolution and the chances of persistence. Efficiency in a network-entity comes from the relative confrontation of the "imaginary values" in each node with absolute and fair references and from the strong and trusted connections within the neighbor nodes. In addition, it is important to understand that any strong disequilibrium in the sharing of resources within any complex network should be avoided as will result, over the wider time scales, as inefficient and even potentially damaging.

From these general principles, we should consider a more efficient and sustainable human network, rediscovering empathy as a possible interconnecting code towards a truly global society, fair in the sharing of resources and in better equilibrium with the surrounding biosphere.

We should avoid inequalities in wealth, health, education, rights and chances of integration and free realization of the proper potential. Having the same chances for every newborn might mean that every "personal wealth" accumulated in a lifetime should be passed to the whole community upon death, to be invested in a social system able to grant fair opportunities to everybody. To survive, eventually, we would need as well to follow common best practices for reducing our environmental footprint, and to collaborate for a more sustainable and distributed production and storage of both energy and food.

If we had to evaluate Fermi's paradox given the experience as human beings on Earth, we could hypothize that maybe "they're still untechnological or just all gone"... Maybe, every intelligent form of life that reached the ability to control and act at planetary scale, in a shortsighted search for personal advantage beyond any equilibrium, collapsed on itself with all its societies and economies, its art and history, stories and emotions, as if so much accumulated power and knowledge and so many wild possibilities could be imagined but not managed. Maybe, the actual "Great Filter of evolution" is just the selfish Ego of beings that pretend to be very smart, but eventually are not so intelligent or really sapiens.

The philosophy introduced starts from a parallel with a possible scientific description of the fundamental "behaviors of a universe" and, following the insights derived in the model, focuses on the efficiencies that should be considered for a sustainable evolution. Being efficient as a truly global human network is not presented as a moral or religious imperative, but as a requirement for persistence. Beyond any recipe for the survival of the homo sapiens species, the author would like to promote the importance of the present awareness and of the consistent sizing of the phenomena, the value of curiosity and openness towards new experiences and connections and, eventually, the need for empathy, in a sense of unity with "the unbroken wholeness of the entire Universe". 
The following table summarizes the parallel between the physical model proposed in the scientific contribution and the philosophical interpretation derived in this paper.

\begin{tabular}{|c|c|c|}
\hline & physical & philosophical \\
\hline \multirow{2}{*}{ present } & $\begin{array}{l}\text { time is emergent as only } \\
\text { the current instant exists }\end{array}$ & $\begin{array}{l}\text { every instant is a unique } \\
\text { present... live it fully! }\end{array}$ \\
\hline & $\begin{array}{l}\text { space is imaginary and emerges } \\
\text { in each instant with max entropy }\end{array}$ & $\begin{array}{l}\text { be imaginative and maximise } \\
\text { experiences and possibilities }\end{array}$ \\
\hline \multirow{2}{*}{ memory } & $\begin{array}{l}\text { spacetime is a real potential } \\
\text { in the Absolute Time memory }\end{array}$ & $\begin{array}{l}\text { consciousness emerges from a logical } \\
\text { consistent access to a memory }\end{array}$ \\
\hline & $\begin{array}{l}E R=E P R=C T C @ T k \\
\quad=\text { memory loops }\end{array}$ & $\begin{array}{l}\text { we are the living network } \\
\text { of our connections }\end{array}$ \\
\hline \multirow{2}{*}{$\begin{array}{l}\text { common } \\
\text { beat }\end{array}$} & $\begin{array}{l}\text { persistence is in the memory } \\
\text { of a common tempo }\end{array}$ & $\begin{array}{l}\text { connections are the sharing } \\
\text { of a common code }\end{array}$ \\
\hline & $\begin{array}{c}\text { the unbroken wholeness of the entire } \\
\text { Universe starts in the firsTk1ss }\end{array}$ & $\begin{array}{l}\text { losing the ego for a greater } \\
\text { sense of wholeness and community }\end{array}$ \\
\hline \multirow{2}{*}{ evolution } & $\begin{array}{l}\text { a quantum energy from the study } \\
\text { of the proper beat of particles }\end{array}$ & $\begin{array}{l}\text { a fair global human society based } \\
\text { on empathy, trust and collaboration }\end{array}$ \\
\hline & \multicolumn{2}{|c|}{... could it be possible? } \\
\hline
\end{tabular}




\section{Annex - A golden lesson}

The golden ratio $\varphi$ is often related to the concept of beauty and equilibrium. It could be found in nature and has inspired several artistic creations, from architecture to music. The idea of beauty is anyway an abstract and subjective concept at human level of abstraction and there are actually infinite ways to describe beauty and justify it with a kind of "mathematical origin", as beauty is often in the eye of the beholder.

There are several ways in which $\varphi$ could be derived. The ancient Greeks described it as the division of a line into "extreme and mean ratio" and used it in several buildings and monuments. Mathematics of the XVI century connected $\varphi$ to the ratio of consecutives "Fibonacci numbers", a series that sum the previous two numbers to get the next one: 1, 1, $2,3,5,8,13,21,34,55 \ldots$ (as the evolution of a memory computing the future from an even contribution of the past and the present or, equivalently, that considers the present as a difference between future and past). Finally, $\varphi$ could be derived from the "continued fraction" that always adds the smallest extra and, in this sense, it is the most irrational number by definition. Actually, it cannot be expressed as a ratio of integers, it is the most irrational due to the limited new information obtained in the progressive truncation of the continued fraction and digits appear as a random sequence.

\section{Euclidean Geometry}



Fibonacci numbers



Continued fraction

$$
\begin{gathered}
\varphi=1+\frac{1}{1+\frac{1}{1+\frac{1}{1+\hookrightarrow}}} \\
\varphi^{2}=\varphi+1 \\
\frac{1}{\varphi}=\varphi-1
\end{gathered}
$$

We could ask if there is any characteristic of the golden ratio that could justify its appearance in nature (even if less often ${ }^{3}$ than what is commonly believed), and then try to understand which kind of "beauty" is achieved by nature in the use of it.

Sunflowers, as well as pinecones, pineapples, and other plants, show series of spirals related to consecutive Fibonacci numbers. This has been proven to be an efficient way to arrange seeds or leaves, as it leads to an optimal "packing per surface" and allows the maximum absorption of sunlight. Plants do not "calculate" continued fractions, but seeds and leaves emerge following the most repetitive and efficient pattern to avoid shading the old ones. This is achieved having a new leaf at an angle of approximately 137.5 degrees from the old one (golden angle, connected to the irrationality of $\varphi$ ).

It seems that "natural beauty" is in the maximum efficiency achieved through the most irrational perspective, derived from an evolving memory that considers the future leveraging on both past and present.

\footnotetext{
${ }^{3}$ nautilus shells and hurricanes for example are simply log-spiral and do not have any relation with the golden ratio
} 


\section{Annex - Pictures from Fantasia}

The conjectures proposed in the paper "Implementation model of a universe based on information" consider spacetime as emerging through imaginary distances from the information stored in the radius of a Black Hole. The model could be referred as Fantasia, a tribute to the Universe where everything could be possible.

The following pictures illustrates the main concepts considered.

An imaginary universe inside a Black Hole
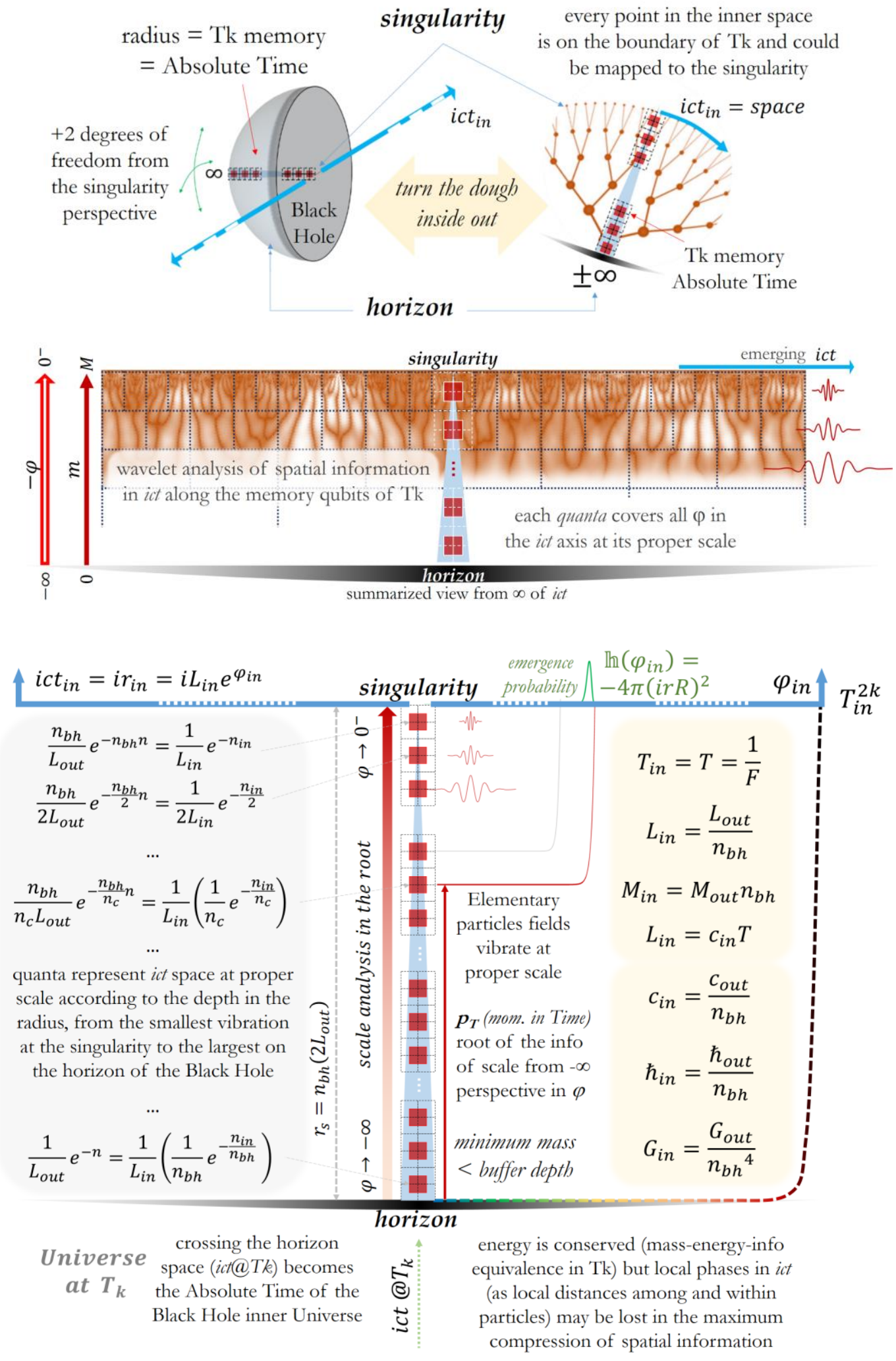
Memory loops in the Absolute Time

each memory quanta has both perspectives at proper scale

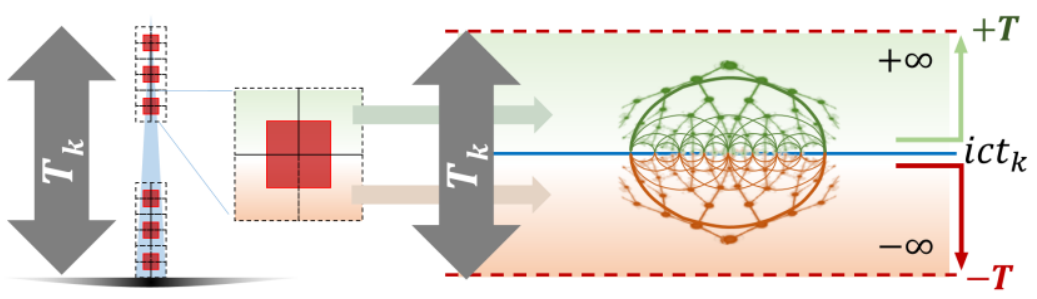

Elementary particles as a network of memory loops in Tk

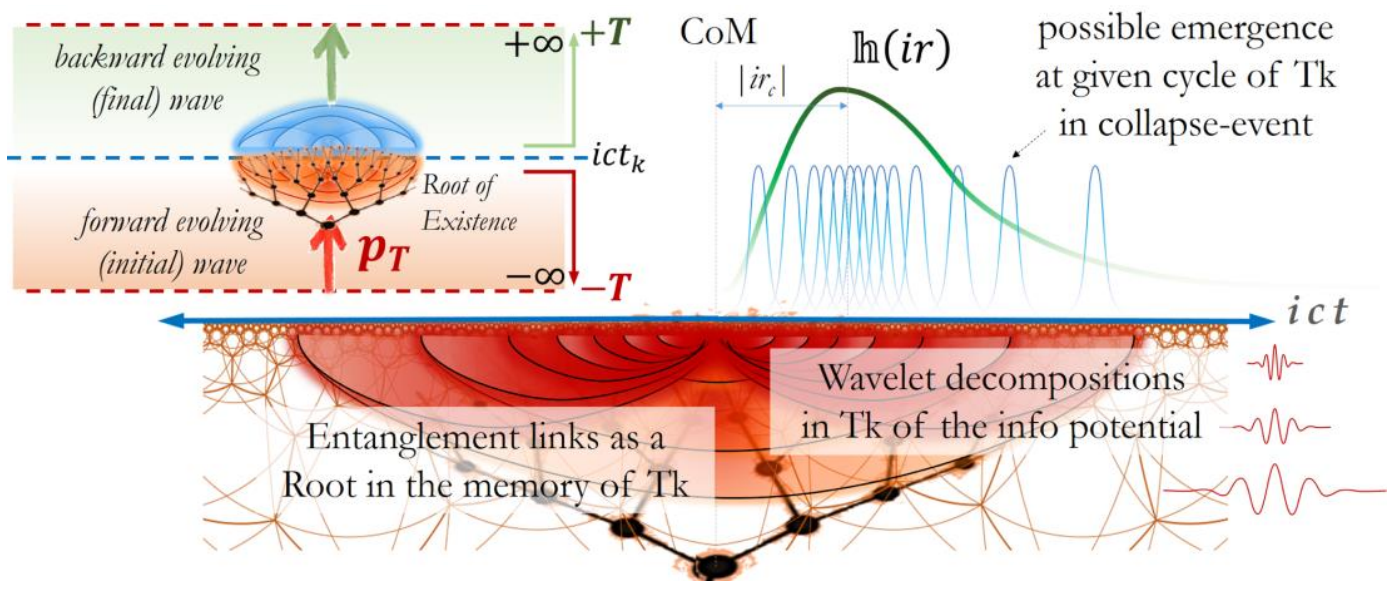

The thick present of a Parent Universe containing a Black Hole




The Arrows of Time

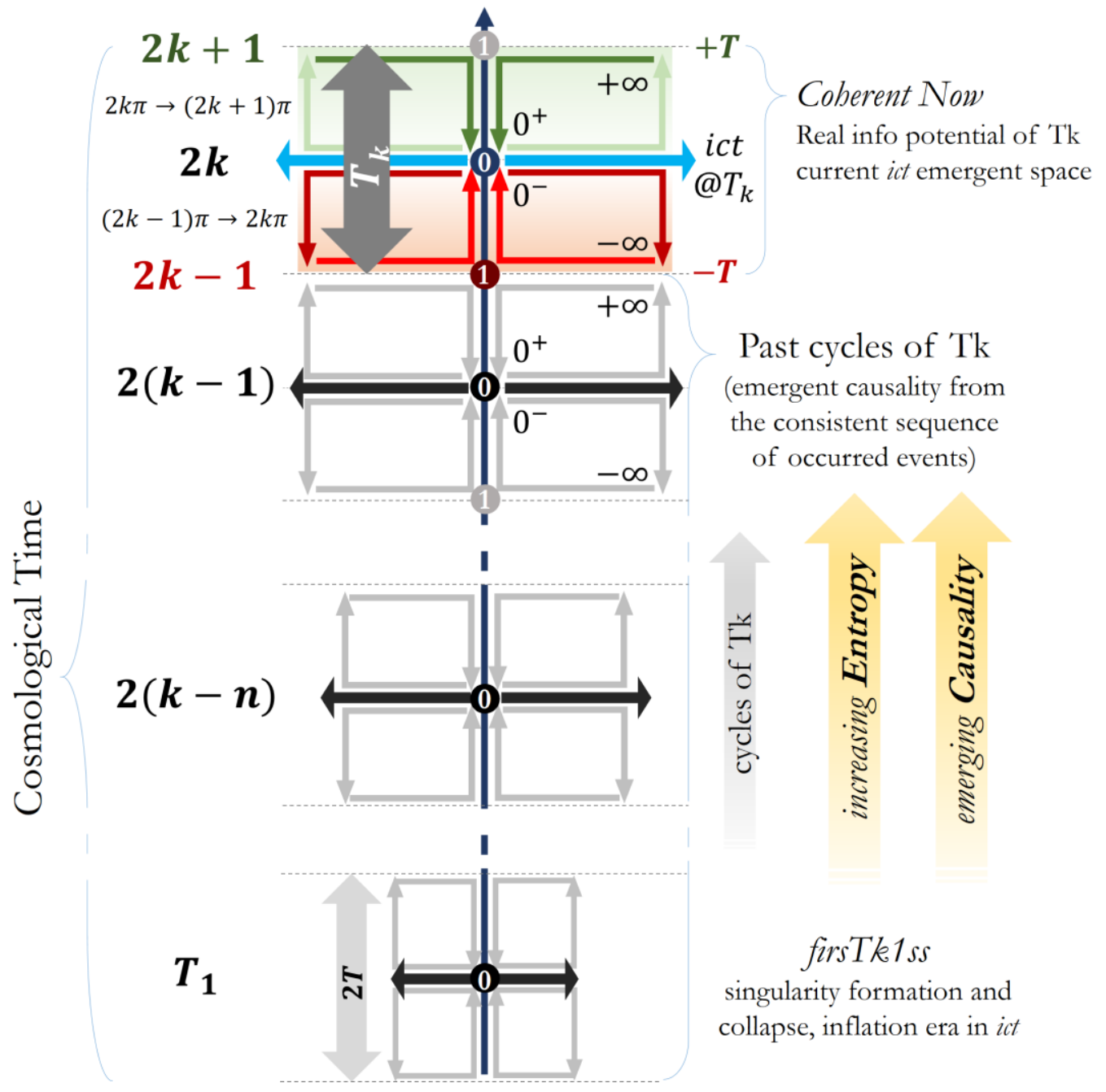

Expansion as a quantum oscillator

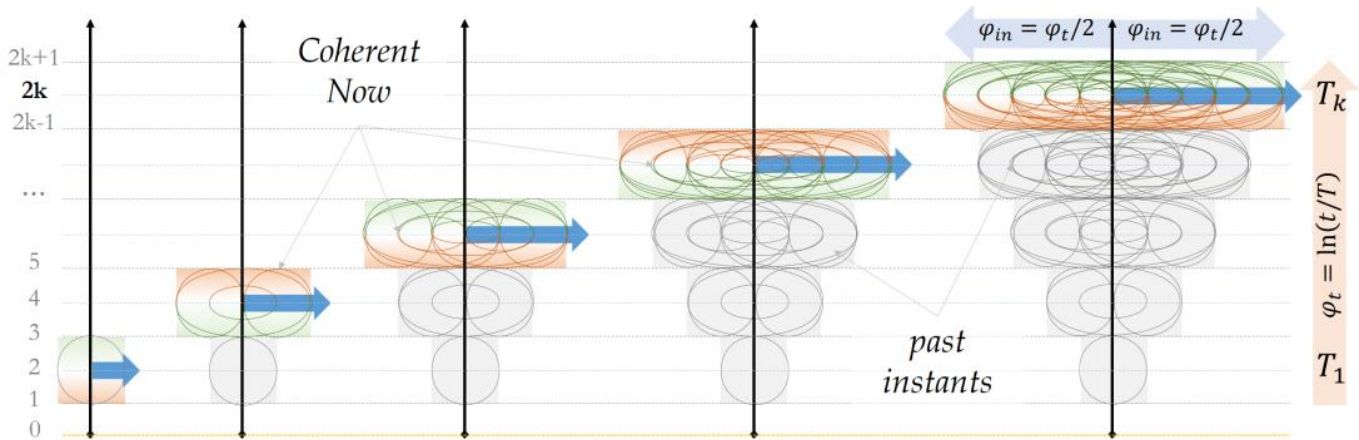

